\title{
Noninvasive Magnetic Resonance Imaging Detection of Cerebral Amyloid Angiopathy-Related Microvascular Alterations Using Superparamagnetic Iron Oxide Particles in APP Transgenic Mouse Models of Alzheimer's Disease: Application to Passive $A \beta$ Immunotherapy
}

\author{
Nicolau Beckmann, ${ }^{1}$ Christelle Gérard, ${ }^{1}$ Dorothée Abramowski, ${ }^{2}$ Catherine Cannet, ${ }^{1}$ and Matthias Staufenbiel ${ }^{2}$ \\ ${ }^{1}$ Global Imaging Group and ${ }^{2 N}$ euroscience Discovery, Novartis Institutes for BioMedical Research, $\mathrm{CH}-4056$ Basel, Switzerland
}

Cerebral amyloid angiopathy (CAA) is a common feature of Alzheimer's disease (AD). More advanced stages are accompanied by microhemorrhages and vasculitis. Peripheral blood-borne macrophages are intimately linked to cerebrovascular pathology coincident with AD. Magnetic resonance imaging (MRI) was used to noninvasively study microvascular lesions in amyloid precursor protein transgenic mouse AD models. Foci of signal attenuation were detected in cortical and thalamic brain regions of aged APP23 mice. Their strength and number was considerably enhanced by intravenous administration of iron oxide nanoparticles, which are taken up by macrophages through absorptive endocytosis, $24 \mathrm{~h}$ before image acquisition. The number of cortical sites displaying signal attenuation increased with age. Histology at these sites demonstrated the presence of iron-containing macrophages in the vicinity of CAA-affected blood vessels. A fraction of the sites additionally showed thickened vessel walls and vasculitis. Consistent with the visualization of CAA-associated lesions, MRI detected a much smaller number of attenuated signal sites in APP23xPS45 mice, for which a strong presenilin mutation caused a shift toward amyloid $\beta_{42}$, thus reducing vascular amyloid. Similar results were obtained with APP24 and APP51 mice, which develop significantly less CAA and microvascular pathology than APP23. In a longitudinal study, we noninvasively demonstrated the reinforced formation of microvascular pathology during passive amyloid $\beta$ immunotherapy of APP 23 mice. Histology confirmed that foci of signal attenuation reflected an increase in CAA-related lesions. Our data demonstrate that MRI has the sensitivity to noninvasively monitor the development of vascular pathology and its possible enhancement by amyloid $\beta$ immunotherapy in transgenic mice modeling AD.

\section{Introduction}

The pathology of Alzheimer's disease (AD) is characterized by amyloid $\beta(A \beta)$-peptide-containing plaques, neurofibrillary tangles consisting of hyperphosphorylated tau, extensive neuritic and synaptic degeneration, and distinct neuron loss. Vascular abnormalities coexist with these $\mathrm{AD}$ features. At autopsy, $\sim 80 \%$ of the $\mathrm{AD}$ patients exhibit cerebral amyloid angiopathy (CAA) (Jellinger, 2002; Greenberg et al., 2004), characterized by deposition of $A \beta$ in the walls of cerebral vessels. The contribution of CAA to the onset of dementia remains unknown. Advanced CAA leads to degeneration of the smooth muscle cell layer, fibrinoid necrosis, and microaneurisms, while it may also occlude capillaries (Thal et al., 2008). Although relatively rare, CAA is associated with hemorrhages ranging from microbleeds to stroke, and with vasculitis presenting a variable degree of lymphocyte infiltration

Received Sept. 21, 2010; revised Nov. 1, 2010; accepted Nov. 8, 2010.

Correspondence should be addressed to PD Dr. Nicolau Beckmann, Global Imaging Group, Novartis Institutes for BioMedical Research, Forum 1, Novartis Campus, WSJ-386.2.09, CH-4056 Basel, Switzerland. E-mail: nicolau.beckmann@novartis.com.

DOI:10.1523/JNEUROSCI.4936-10.2011

Copyright $\odot 2011$ the authors $\quad 0270-6474 / 11 / 311023-09 \$ 15.00 / 0$ and vascular thickening (Vinters et al., 1998; Kinnecom et al., 2007). Both conditions are accompanied by the presence of macrophages/ microglia, which surround affected vessels (Maat-Schieman et al., 1997; Vinters et al., 1998). Microbleeds, also referred to as microhemorrhages, have long been considered clinically silent. More recently, their potential contribution to the development of cognitive dysfunction or as a risk factor of hemorrhagic stroke has been extensively investigated (Cordonnier et al., 2007).

Detecting such events in vivo can help to better understand the role of CAA in the development of dementia as well as in complications potentially related to $\mathrm{A} \beta$ immunization, a therapeutic approach that has been the subject of intense investigation [see Brody and Holtzman (2008) for a recent review]. In animal models, $A \beta$ immunotherapy has been shown to reduce parenchymal amyloid, while it may increase cerebral microhemorrhages associated with amyloid-laden vessels (Pfeifer et al., 2002; Wilcock et al., 2007). A recent histological follow-up study of the first active $A \beta$ immunotherapy in humans also suggested an increase in CAA and associated microhemorrhages (Boche et al., 2008). These results hint at a link between an increase in CAA and possible complications of $\mathrm{A} \beta$ immunization. 
Following intravenous administration, superparamagnetic iron oxide (SPIO) particles are taken up by macrophages through absorptive endocytosis (Weissleder et al., 1990). Macrophages loaded with iron particles present in damaged areas can lead to local signal changes in magnetic resonance (MR) images, because of magnetic susceptibility effects of the iron. This property has been explored to follow with MR imaging (MRI) the infiltration of macrophages into inflamed areas in several disease models in rodents and in patients [for a recent review, see (Beckmann et al., 2009)].

In this work, we have used APP23 transgenic mice (Sturchler-Pierrat et al., 1997), which develop a considerable amount of vascular (CAA) and parenchymal (plaque) amyloid, to demonstrate the feasibility of detecting CAA-related microvascular lesions by MRI in combination with SPIO administration. The specificity of the method was further shown by comparison with three additional amyloid precursor protein (APP) transgenic lines differing in the relative amount of CAA and compact or diffuse parenchymal amyloid plaques. Moreover, we demonstrated the suitability of this imaging approach to detect and longitudinally follow vascular alterations induced by passive $\mathrm{A} \beta$ immunotherapy of APP23 mice.

\section{Materials and Methods \\ Animals}

All transgenic mice used expressed the transgene under control of a murine Thy- 1 promoter element. The generation of APP23 [human APP with the K670N/M671L mutation (SturchlerPierrat et al., 1997)], APP24 [human APP with the K670N/M671L and V717L mutations (Abramowski et al., 2008)], APP51 [human wildtype APP (Bodendorf et al., 2002)], and PS45 [human presenilin-1 with the G384A mutation (Herzig et al., 2004)] mice has been described earlier. APP23xPS45 were obtained by crossbreeding the respective lines (Busche et al., 2008). All mice were hemizygous for the transgene(s) of interest and were bred in-house. Male and female APP23, male APP24, female APP51, male APP23xPS45, and age- and gender-matched wild-type mice have been used throughout the study as indicated in the figure legends.

Animals were housed under standard conditions (temperature $20-24^{\circ} \mathrm{C}$, relative humidity minimum $40 \%$, light/dark cycle $12 \mathrm{~h}$ ) and fed a standard diet (Kliba Nr. 3893.025, Frantschach, Rothrist) and water supply ad libitum. Mice of different ages were measured by MRI. Age-matched nontransgenic littermate mice served as controls. Animal handling, care, and experimental use were in line with the Swiss federal law for animal protection. The study has been approved by the Veterinary Department of the City of Basel (animal licenses BS-1094 and -1283).

\section{Passive immunization}

Male mice were passively immunized weekly by intraperitoneal injections of $0.5 \mathrm{mg}$ of $\beta 1$ mouse monoclonal IgG2a antibody that recognizes amino acids 3-6 of human A $\beta$ (Paganetti et al., 1996). Age-matched control mice received weekly intraperitoneal injections of $0.5 \mathrm{mg}$ of a

B
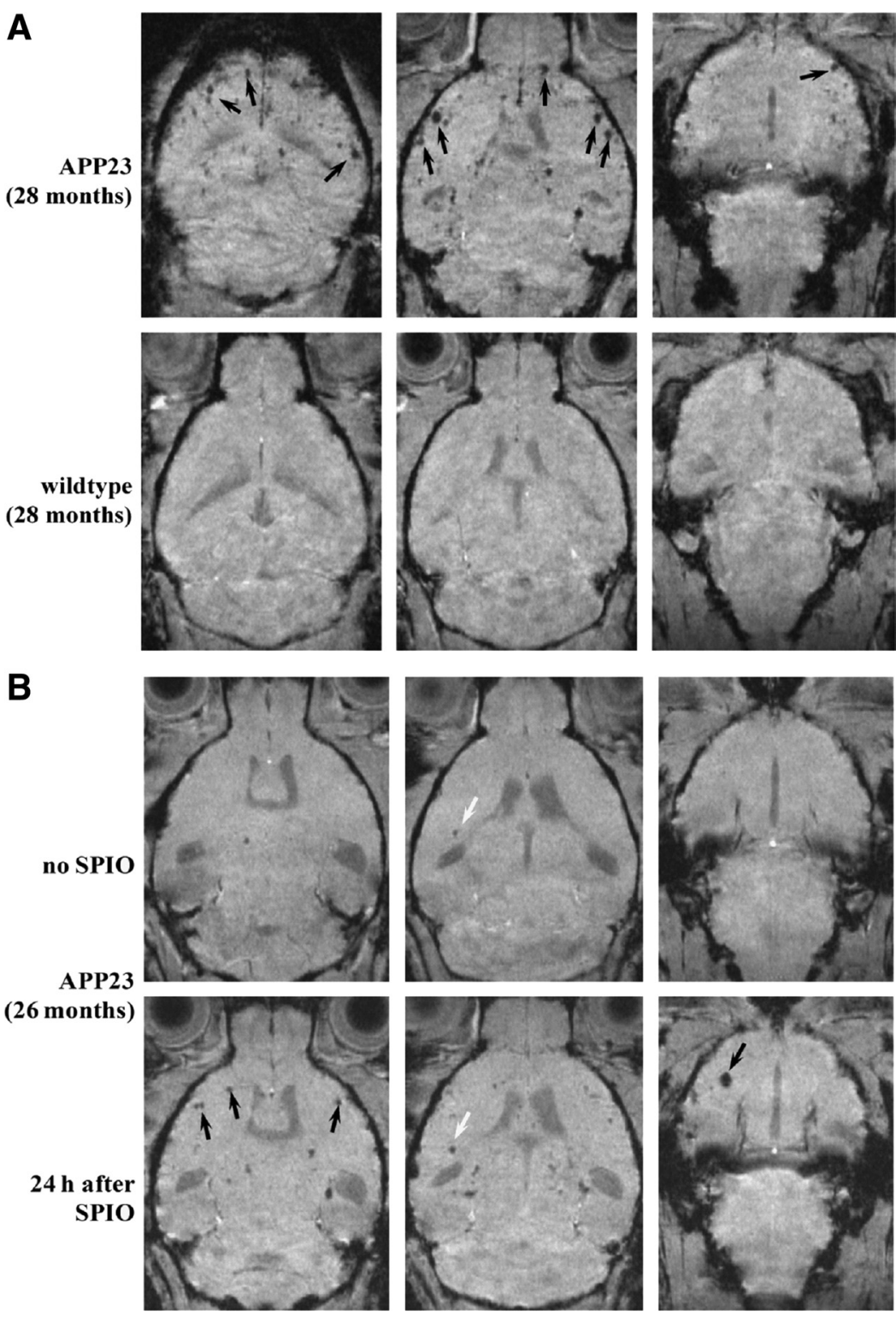

Figure 1. MRI from old male mice. $A$, Representative images extracted from 3D datasets acquired from 28 -month-old mice $24 \mathrm{~h}$ after SPI0 administration. Sites of signal attenuation (arrows) were detected throughout the cerebral cortex of the APP23, but not in the wild-type mouse. $\boldsymbol{B}$, Images from a 26-month-old APP23 mouse, extracted from a 3D dataset acquired before (upper row) and $24 \mathrm{~h}$ after SPIO administration (lower row). The interval between both measurements was 2 days. Before SPI0, one site of signal attenuation was encountered in the cortex (white arrow). This site became more prominent following SPIO. In addition, many other foci of signal attenuation became apparent (black arrows).

control antibody (monoclonal mouse anti-wheat auxin IgG2a antibody; AMS Biotechnology). Four groups of mice were formed: (1) APP23 mice immunized with the $\beta 1$ antibody ( $n=9$ at the beginning of the immunization); (2) APP23 mice immunized with the control antibody ( $n=10$ at the beginning of the immunization); (3) wild-type mice immunized with the $\beta 1$ antibody ( $n=10$ at the beginning of the immunization); (4) wild-type mice immunized with the control antibody ( $n=10$ at the beginning of the immunization). All animals were 18 months old at the beginning of the immunization.

\section{MRI}

For the MRI investigations, animals were anesthetized with $1.3 \%$ isoflurane (Abbott) in a mixture of oxygen/ $\mathrm{N}_{2} \mathrm{O}(1: 2)$ administered via a face mask. The body temperature of the mice was kept at $37^{\circ} \mathrm{C}$. No stereotactic holding was used.

Measurements were performed with a Biospec $47 / 40$ spectrometer (Bruker Medical Systems) operating at $4.7 \mathrm{~T}$, equipped with an actively 


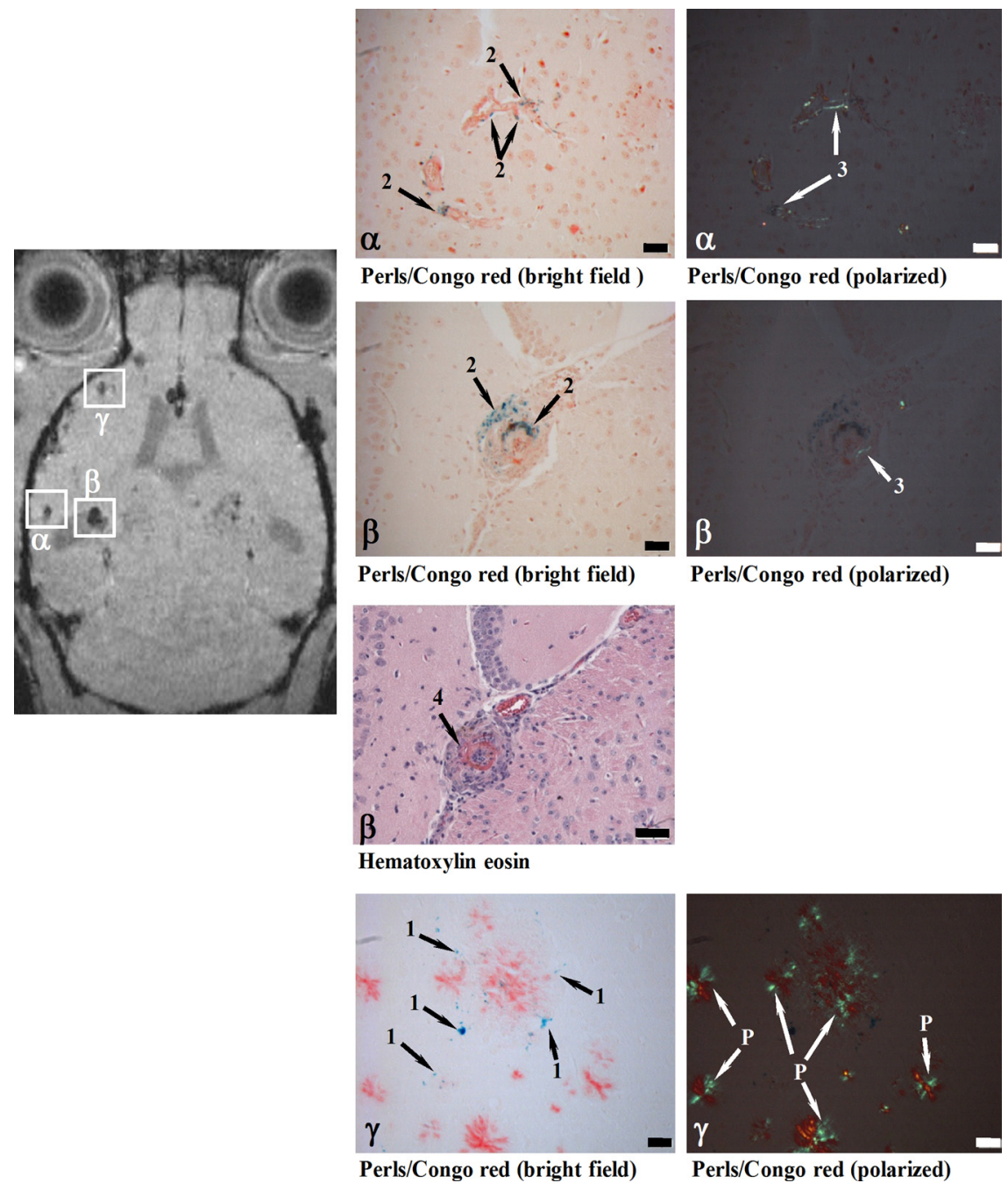

Figure 2. Histological examination of cerebral cortex sites with foci of attenuated MRI signal $(\alpha, \beta, \gamma)$. At $24 \mathrm{~h}$ following SPIO administration, a male 28-month-old APP23 mouse was analyzed in vivo by MRI and processed for histology immediately thereafter. Perls/Prussian blue staining showed iron-loaded macrophages in CAA-laden vessels (Congo red positive) at both sites ( $\alpha$ and $\beta$ ). While the vessel walls were thickened at both $\alpha$ and $\beta$ locations, only site $\beta$ in addition showed vasculitis characterized by lymphocyte infiltration (Hematoxylin eosin). At site $\gamma$, isolated iron-loaded macrophages (severity grade 1 ) were present close to amyloid vessels. 1, Iron in isolated macrophages; 2 , iron in macrophages at the vessel wall; 3 , amyloid deposit in vessel wall; 4 , vasculitis; $\mathrm{P}$, amyloid plaque. Scale bars, $50 \mu \mathrm{m}$. Congo red-stained sections were observed under bright field or polarized light.

shielded gradient system. The operational software of the scanner was Paravision (Bruker). Images were obtained using a three-dimensional (3D) gradient-echo sequence with the following imaging parameters: repetition time $40 \mathrm{~ms}$; echo time $8 \mathrm{~ms}$; matrix $256 \times 192 \times 48$; field-ofview $2.8 \times 1.44 \times 1.44 \mathrm{~cm}^{3}, 2$ averages. Total acquisition time for an image having a pixel size of $109 \times 133 \times 300 \mu \mathrm{m}^{3}$ was of $12.3 \mathrm{~min}$. The datasets were reconstructed to $256^{3}$.

\section{MR image analysis}

Analysis was performed by a blinded investigator. Sites in the cortex presenting signal attenuation with a minimum diameter of $150 \mu \mathrm{m}$ were counted throughout the whole brain. To ensure that the same site was not counted multiple times, its presence was carefully controlled over several consecutive slices from the 3D dataset. In an initial phase, two investigators who were unaware of the genotype and of the age of the mice analyzed fifteen $3 \mathrm{D}$ datasets, with $10-16$ sites of signal attenuation registered for each dataset. The agreement between the observers was $90 \%$.

\section{MRI contrast agent}

Colloid-based SPIO (Endorem, $11.2 \mathrm{mg}$ of Fe/ml; Guerbet) particles, so-called ferumoxides, were used. Ferumoxides represent $\mathrm{Fe}_{2} \mathrm{O}_{3}$ and
$\mathrm{Fe}_{3} \mathrm{O}_{4}$ particles with a mean particle diameter of $150 \mathrm{~nm}$, consisting of nonstoichiometric magnetic crystalline cores covered with a 3-nm-thick dextran T-10 layer (Jung, 1995). Animals received a bolus of Endorem $(0.2 \mathrm{ml})$ intravenously $24 \mathrm{~h}$ before an imaging session. To exclude that the injection of SPIO itself could not have elicited vascular damage, 28 - to 30 -month-old APP23 mice $(n=6)$ were imaged by MRI before and $1 \mathrm{~h}$ after intravenous administration of physiological saline $(0.2 \mathrm{ml})$ as a bolus.

\section{Postmortem analyses}

Mice $(n=40$ male APP23, $n=7$ female APP51, $n=6$ male APP23xPS45, $n=18$ male APP24) were overdosed with pentobarbital. The brains were removed from the skull and fixed for histology, or both hemispheres were separated. One brain hemisphere was then fixed for histology, while the forebrain from the second hemisphere (excluding olfactory bulb, brainstem and cerebellum) was used for assessments of $A \beta$ levels. Analyses were performed blind to the genotype and treatment of the mice. Brains from $n=20 \mathrm{APP} 23$ mice were used solely for histology, and from $n=13$ APP23 and $n=13$ APP2 4 animals for determination of $A \beta$ levels only. Brains from the remaining animals were submitted to both analyses.

Histology. Removed whole or half brains were fixed by immersion in $4 \%$ formaldehyde in $0.1 \mathrm{M} \mathrm{PBS}$ at $4^{\circ} \mathrm{C}$ for 1 week at minimum. After dehydration through increasing graded series of ethylic alcohol $(50 \%, 70 \%, 80 \%$, and $100 \%$ ), brains were trimmed to get horizontal (coronal) sections and embedded in paraffin. Acquisition of 3D MRI datasets facilitated the detection of lesions, as these could be searched for through the whole brain. For the validation, the horizontal (coronal) coordinate of the slice showing a lesion in the MRI served as basis to localize the corresponding lesion by histology. Eight consecutive 5- $\mu$ m-thick sections were cut with a microtome at the horizontal level provided by MRI. Sections were mounted onto Superfrost slides, dried at $37^{\circ} \mathrm{C}$, deparaffinized in xylene, hydrated through graded ethylic alcohol, and washed in deionized water. Hematoxylin and eosin staining according to standard protocols was used to assess the general morphology. The Perls/Prussian blue reaction and the Congo red staining were used respectively to visualize ferric iron and to demonstrate amyloid. Grading of iron-containing microglia was done according to Pfeifer et al. (2002). Severity grades 1 and 2 ( $\leq 10$ cells) were summarized as moderate, in contrast to grade 3 ( $>10$ cells, abundant).

Immunohistochemistry was performed on paraffin sections according to previously published protocols (Calhoun et al., 1999) by using the avidin-biotin-peroxidase complex method (Vector Laboratories) with diaminobenzidine as chromogen. NT12 antiserum raised against $\mathrm{A} \beta_{40}$ (Paganetti et al., 1996) was diluted 1:2000 in PBS with 3\% goat serum and incubated overnight at $4^{\circ} \mathrm{C}$. After rinsing, sections were incubated for $1 \mathrm{~h}$ with biotinylated anti-rabbit IgG secondary antibody (BA1000, Vector Laboratories) diluted 1:200 in PBS. Following another rinsing, sections were further processed with the avidin-biotin-peroxidase technique (ABC-Elite Kit PK6100; Vector Laboratories). Finally, sections were reacted with metal-enhanced diaminobenzidine substrate (Code 1718096, Boehringer), counterstained with hemalum, dehydrated, cleared in $x y-$ lene, and coverslipped. 
Quantification of total $A \beta$ in brain homogenates. Forebrain hemispheres, excluding olfactory bulb, brainstem, and cerebellum, were weighed, homogenized by sonication in 9 volumes of Tris-buffered saline, $20 \mathrm{~mm}$ Tris- $\mathrm{HCl}$, pH 7.6, 137 mm sodium chloride, and protease inhibitor cocktail Complete (Roche Molecular Biochemicals), and extracted with $70 \%$ formic acid. The extracts were neutralized by addition of 19 volumes of $1 \mathrm{M}$ Tris-base and centrifuged for $15 \mathrm{~min}$ at $20,000 \times \mathrm{g}$. The supernatants were analyzed by electrochemoluminescencelinked immunoassays (Human $\mathrm{A} \beta 40$ and A $\beta 42$ Ultrasensitive Kits, Meso Scale Discovery) (Abramowski et al., 2008).

\section{Data analysis}

Mean values ( \pm SEM) from $n$ individual animals are presented. Mann-Whitney analyses were performed on the counts at the different time points with SYSTAT 11 (Systat Software). In addition, for the longitudinal assessment of the effects of immunization, the $\log 10$ of the counts were analyzed using an extension of ANOVA, called "Mixed model analysis" or "ANOVA with random effects" in SYSTAT 12. For multiple comparisons, a Bonferroni correction followed the ANOVA. Significance was assumed at the $5 \%$ probability level.

\section{Results}

\section{Microvascular lesions in APP23 mice} revealed by MRI

The feasibility of detecting CAA-related changes in mice was tested using MRI in the absence and in combination with iron oxide nanoparticles. Optimization of the method with APP23 mice, which develop significant CAA (Calhoun et al., 1999), led to a sensitive $\mathrm{T}_{2}{ }^{*}$-weighted gradient-echo imaging sequence used throughout the present study. Similar to previous experience with MRI detection of macrophage infiltration into inflamed peripheral tis-

sues (Beckmann et al., 2009), an interval of $24 \mathrm{~h}$ between SPIO administration and image acquisition turned out to be well suited. Figure $1 A$ shows coronal MR images extracted from a $3 \mathrm{D}$ dataset acquired from a 28-month-old mouse. Focal signal attenuations were observed throughout the cerebral cortex (indicated by arrows) as well as in thalamic regions. Instead, diffuse contrast changes over an extended region are detected in peripheral tissues with macrophage infiltration or in the brain as shown for stroke and multiple sclerosis [see Beckmann et al., 2009 for a review]. No signal change was detected in the brains of age-matched wildtype controls (Fig. 1A). Although rare, sites of attenuated signal could be detected in cortical areas of APP23 mice without contrast agent injection (Fig. $1 B$ ). After SPIO administration, these sites were more prominent and additional foci of signal attenuation became clearly visible as demonstrated in Figure $1 B$. For comparison, in 28-month-old APP23 mice, the mean number of sites showing MRI signal attenuation in the cortex was $0.5 \pm 0.2$ ( $n=10$ animals $)$ and $10.1 \pm 1.5(n=8$ animals $)$ without and with SPIO administration, respectively.

Histology revealed iron-containing macrophages at the sites of MRI signal attenuation in all SPIO-injected APP23 mice. Figure 2 shows the histological analysis of regions in cerebral cortex

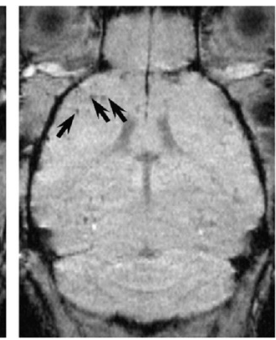

20 months
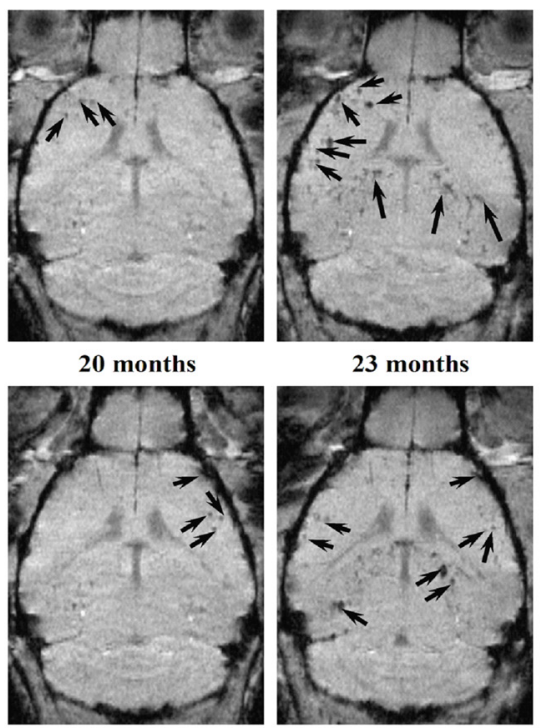

23 months
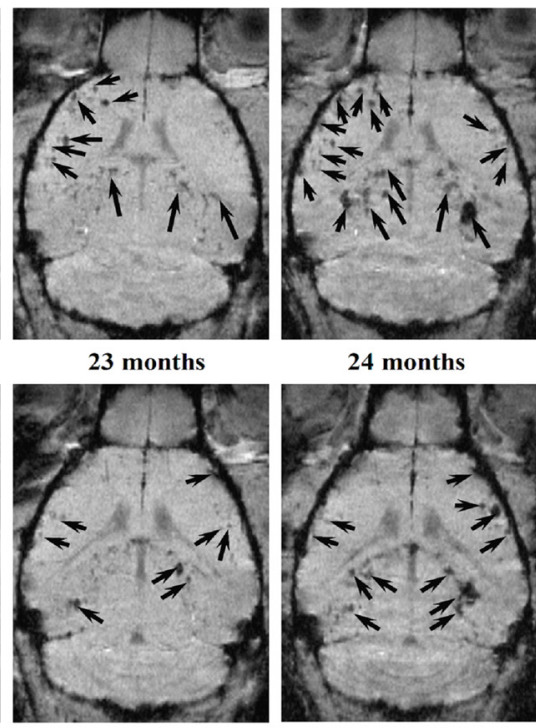

24 months
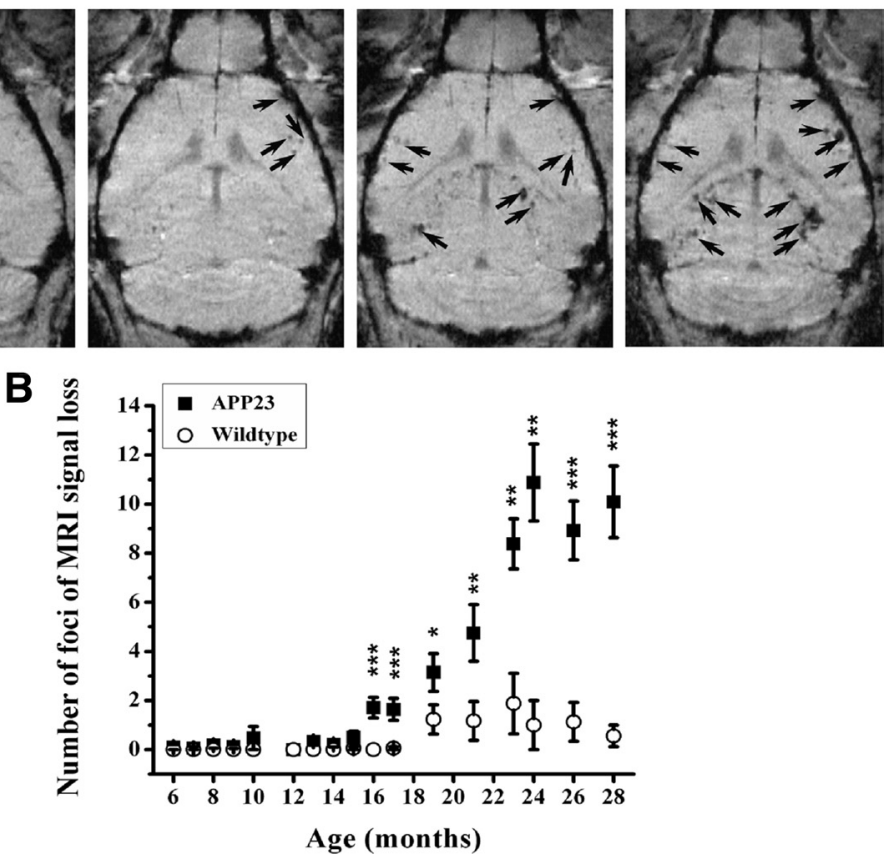

Figure 3. Age-related effects in male APP23 mice. $\boldsymbol{A}$, Foci of attenuated signal are shown in two slices extracted from 3D MRI datasets acquired at different ages from a representative animal. SPIO was administered $24 \mathrm{~h}$ before each image acquisition. $\boldsymbol{B}$, Age-dependent increase in the number of sites presenting signal attenuation in the brain cortex of male APP23 mice and agetistical tests performed between APP23 and wild-type mice at each specified age. The contrast agent, SPI0, was administered

matching three foci of attenuated MRI signal. At two sites, ironcontaining macrophages were found in the wall of thickened vessels, which also contained amyloid deposits. One of the vessels ( $\operatorname{site} \beta$ ) in addition presented vasculitis with typical infiltration of lymphocytes. At the third site, isolated iron-loaded macrophages (severity grade 1) were present close to amyloid-laden vessels. Detailed histological analyses of 60 regions of MRI signal attenuation (five APP23 animals; 26-28 months of age) revealed clusters of iron-containing macrophages of all severity grades (grades 1,2 : moderate: $\leq 10$ cells; grade 3 : abundant: $>10$ cells), mostly in close vicinity to CAA-affected blood vessels. Grade 1 and 2 lesions had sizes up to $250 \mu \mathrm{m}$ in the MR images, whereas grade 3 lesions appeared typically at sizes of $300-400 \mu \mathrm{m}$ in the MRI. No blood vessel association was apparent for a fraction of the moderate sites ( $17 \pm 11 \%$ of sites), but it may have been missed on the thin $5 \mu \mathrm{m}$ sections. In contrast, CAA-affected blood vessels were present at the majority of the sites with moderate $(65 \pm 17 \%)$ and at all sites with abundant $(18 \pm 16 \%)$ iron-containing macrophages. The majority of these cells were located outside the blood vessels. In the walls of CAA-affected vessels, they were relatively rare $(8 \pm 1 \%$ of CAA vessels). Additional cerebrovascular alterations such as a thickened vessel wall and vasculitis were found in 


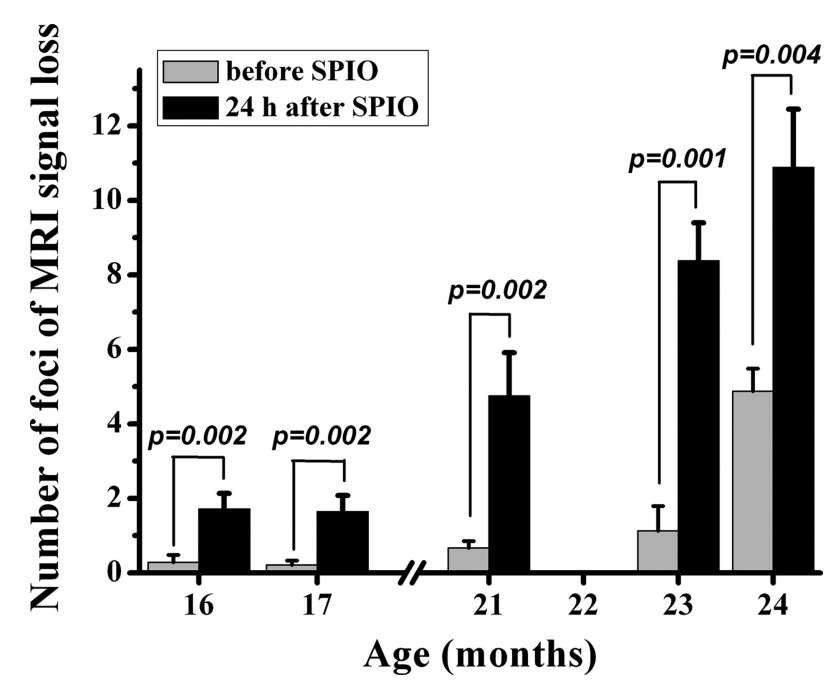

Figure 4. Effect of repeated SPIO administration on number of sites presenting signal attenuation in male APP23 mice. The same animals were repeatedly measured by MRI at the specified ages, just before and $24 \mathrm{~h}$ following SPIO administration. For APP23 mice older than 21 months, some foci of signal loss were detected even 1 or 2 months after the last SPI0 administration. Data are given as mean \pm SEM. The significance levels $p$ refer to Mann-Whitney comparisons.

$\sim 30 \%$ of the sites of MRI signal attenuation. None of the sites showed an acute hemorrhage. Histological analyses of wild-type mice after SPIO administration did not identify any iron or other pathological alteration in the brain consistent with the absence of MRI signal foci. Together these data indicated that the MRI protocol detected a range of microvascular lesions from minor to more severe pathologies.

Brains of three APP23 animals (age 26-28 months) that had not received SPIO were also examined by histology. At sites of MRI signal attenuation, iron-containing macrophages (moderate number) associated with CAA-affected vessels were detected. In addition, a thickened vessel wall and vasculitis were detected at these sites. Iron-containing macrophages were only rarely found in the vessel wall ( $1 \pm 0.8 \%$ of analyzed CAA vessels).

To investigate whether the injection of SPIO itself might elicit vascular damage, APP23 male mice $(n=6)$ 28-30 months of age were measured by MRI before and $1 \mathrm{~h}$ after intravenous injection of saline as a bolus. No additional sites of signal attenuation were observed following saline administration (data not shown). Moreover, a careful histological analysis of 28- to 30-month-old APP23 mice did not reveal increased signs of inflammation or CAA pathology in animals that received SPIO. These observations demonstrate that the injection of SPIO per se did not cause vascular damage.

\section{Age-dependent increase in microvascular lesions in APP23 mice}

The number of pathological blood vessels increases with age and amyloid deposition in APP23 transgenic mice (Winkler et al., 2001). Consistently, we found an age-dependent elevation of attenuated signal foci in the cerebral cortex of male APP23 mice that had received SPIO before MRI (Fig. 3). The number of sites displaying signal attenuation increased from 16 months of age onwards. In wild-type controls, only a very small increase in number of foci was observed after an age of 18 months. The number of sites displaying signal loss in the cortex of female transgenic mice developed in a similar way and was not significantly different from that in male APP23 mice.
A
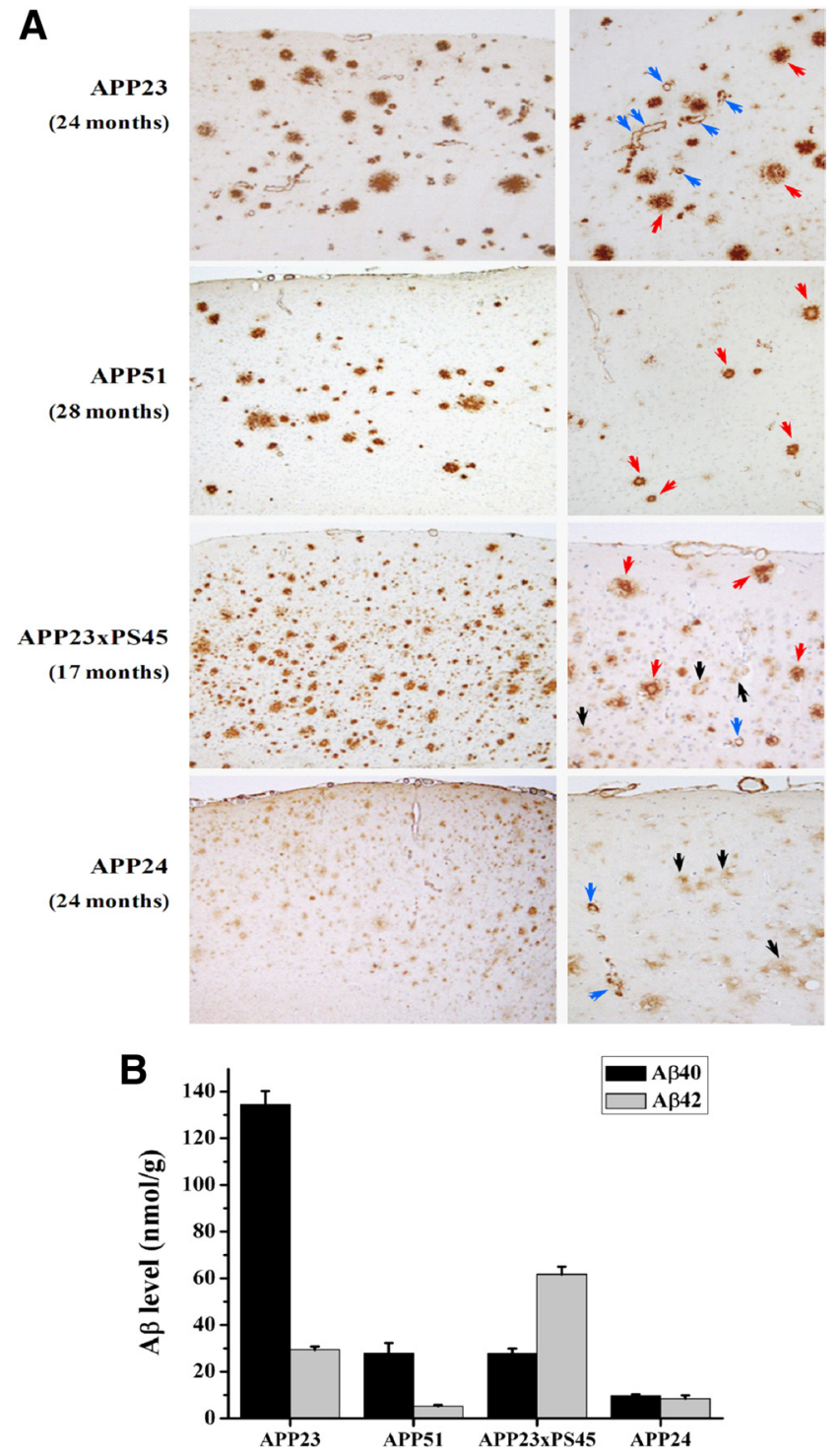

Figure 5. Characterization of various transgenic mouse lines differing by the relative amount of CAA and parenchymal amyloid plaques. $\boldsymbol{A}$, Histological sections were stained with a rabbit polyclonal antibody to $A \beta$ (NT12) indicating the presence of amyloid plaques (red arrows), diffuse plaques (black arrows), and vascular amyloid (blue arrows). Note the difference in CAA frequency as well as severity between the lines. $\boldsymbol{B}$, Forebrain concentrations (means \pm SEM) of $A \beta_{40}$ and $A \beta_{42}$ for the different APP transgenic mouse lines at the ages used: APP23 (24- to 28-month-old male mice, $n=20$ ), APP51 (28-month-old female mice, $n=7$ ), APP23xPS45 (13- to 17-month-old male mice, $n=6$ ), and APP24 (26-month-old male mice, $n=18)$ animals.

We next examined the long-term stability of the signal attenuation following SPIO injection. Animals labeled with SPIO at 16 months completely lost the signal when reanalyzed 1 month later. In contrast, for APP23 mice older than 23 months, sites presenting signal attenuation were visible even 1 or 2 months after SPIO administration (Fig. 4). Therefore, we verified whether repeated administration of SPIO might have led to different results compared to a single dose. Similar numbers of foci were encountered in 28-month-old APP23 mice that had received one or multiple injections of SPIO $[10.0 \pm 2.7$ foci (means \pm SEM) for a single injection of SPIO to $n=5$ mice; $10.2 \pm 1.7$ foci for seven administrations of SPIO to 6 mice, starting at an age of 19 months, with a minimum interval of 1 month between consecutive contrast agent administrations], demonstrating that any re- 

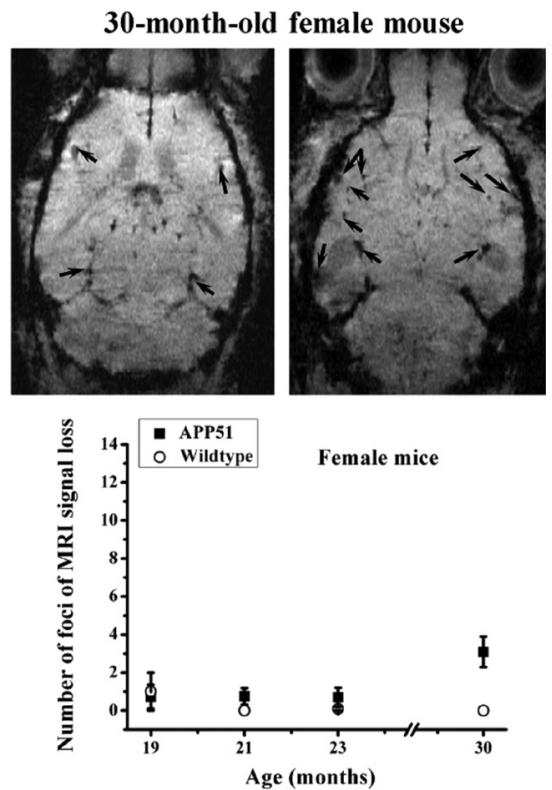
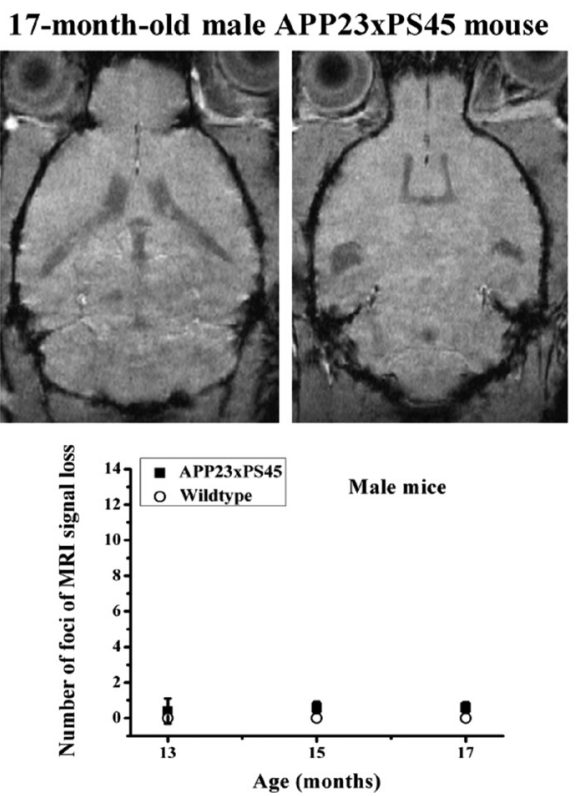
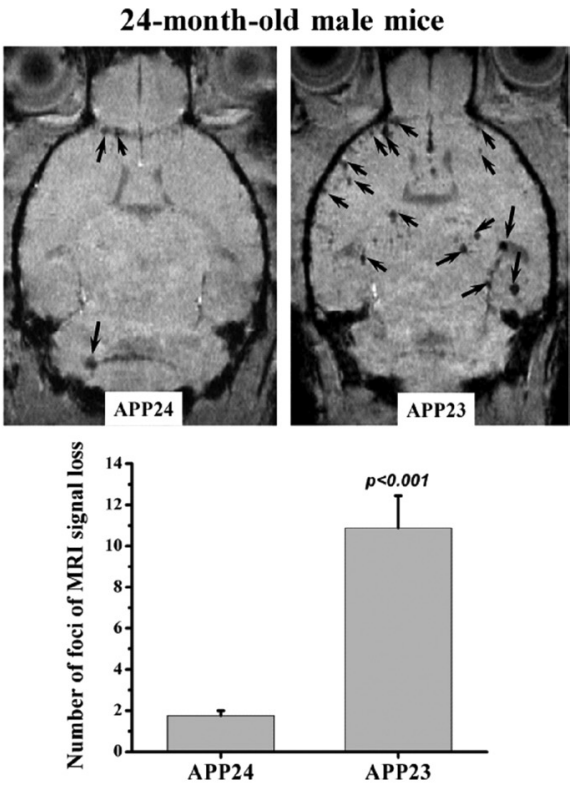

Figure 6. Comparison of the different APP transgenic mouse lines for foci of MRI signal attenuation in the cerebral cortex. Representative images from each line are presented with corresponding signal attenuation foci indicated by arrows. The number of sites is given as means \pm SEM. Five to twelve animals were analyzed per group and time point. The level of significance $p$ refers to Mann-Whitney statistical comparisons. Images were acquired $24 \mathrm{~h}$ after SPIO injection.

sidual label did not affect the total number of sites detected after acute SPIO administration.

\section{MRI analyses of APP transgenic mouse lines differing in cerebral amyloid angiopathy}

To further evaluate the specificity of the method for microvascular lesions, these changes were reduced by crossbreeding APP23 with mutated presenilin transgenic mice (APP23xPS45, analyzed at 15-17 months). We also used transgenic lines forming less CAA as they express human wild-type APP (APP51 mice, 28-30 months) or APP with the Swedish and London mutations (APP24 mice, 24 months). Histological analysis of the neocortex (Fig. $5 A$ ) confirmed that vascular $\mathrm{A} \beta$ was most pronounced in 24- to 28-month-old APP23 mice. Most parenchymal amyloid plaques were compact, but a considerable amount of diffuse deposits were present as well at this advanced age. In contrast, only scattered vascular amyloid was seen in APP23xPS45 mice, although they contained as much compact and more diffuse $\mathrm{A} \beta$ deposits at this younger age (Fig. 5A). Biochemically this histological change was associated with a shift of $A \beta$ isoforms from $\mathrm{A} \beta_{40}$ to $\mathrm{A} \beta_{42}$ (Fig. $5 B$ ) as expected (Herzig et al., 2004; Busche et al., 2008). APP51 mice showed a similar distribution of the different forms of $\mathrm{A} \beta$ deposits as APP23, but the deposits were generally less abundant (Fig. 5A). This was reflected in the lower $\mathrm{A} \beta$ level but an unchanged $\mathrm{A} \beta_{42} / 40$ ratio (Fig. $5 B$ ). For APP24 mice, $A \beta$ deposits in the cerebral cortex were predominantly diffuse. To a lesser degree, compact amyloid plaques could also be found. The amount of vascular A $\beta$ was lower than that in APP23 animals (Fig. $5 A$ ). In agreement, the $A \beta$ level was generally lower than in APP23 mice and the shift from $A \beta_{40}$ to $A \beta_{42}$ was moderate compared to APP23xPS45 (Fig. 5B).

In contrast to the MRI results obtained with APP23 animals, no significant increases in number of sites displaying MRI signal attenuation were found in the cortex or other brain regions of APP23xPS45, APP24, and APP51 transgenic mice (Fig. 6). A minor, nonsignificant increase may have occurred in APP51 mice at 30 months of age. These observations are consistent with the
Table 1. Comparison of the different forms of $A \boldsymbol{\beta}$ deposition in the cerebral cortex of the four transgenic lines (5-7 brains per line, 3 slices per brain) used in the present study

\begin{tabular}{llll}
\hline Transgenic line/age & $\begin{array}{l}\text { Compact amyloid } \\
\text { plaques }\end{array}$ & Diffuse plaques & $\begin{array}{l}\text { Vascular amyloid } \\
\text { (CAA) }\end{array}$ \\
\hline APP23 (24 months) & ++++ & ++ & +++ \\
APP51 (28-30 months) & +++ & ++ & ++ \\
APP23XPS45 (15-17 months) & ++++ & +++ & + \\
APP24 (24 months) & ++ & +++ & ++
\end{tabular}

The total number of amyloid plaques and vascular amyloid profiles per slice was estimated and classified with the following grading system:,$+ 5-10$ deposits;,$++ 11-50$ deposits;,$+++ 51-100$ deposits; ++++ , more than 100 deposits. All animals were male except for the APP51 mice.

histological results presented above and summarized in Table 1, indicating a more pronounced presence of vascular A $\beta$ in APP23 than in the other three lines.

\section{MRI detection of increased microvascular lesions following passive A $\beta$ immunotherapy of APP23 mice}

Treatment of plaque-bearing APP23 mice with $A \beta$ antibody $\beta 1$ was demonstrated to increase microhemorrhages (Pfeifer et al., 2002). We have used a similar passive immunization protocol to determine whether the MRI paradigm developed was able to detect these microvascular lesions in vivo. Starting at 18 months of age, APP23 mice received either $0.5 \mathrm{mg}$ of $\beta 1$ antibody (recognizing amino acids 3-6 of $A \beta$ ) or unrelated control antibody and were imaged at baseline and at different time points during the treatment. The number of foci presenting signal attenuation in the cerebral cortex increased during $\beta 1$ immunization (Fig. 7). In contrast, there was only a small and nonsignificant trend toward an increase in the control antibody-treated animals. No sites of signal attenuation were detected in the brains of age-matched wild-type mice that received either the $\beta 1$ or the control antibody. Histology performed at the end of the study showed a significant increase of blood vessels associated with CAA and of iron-containing macrophages in the cerebral cortex of $\beta 1$ antibody-treated APP23 mice (Table 2). This confirms the described increase in CAA-related microvascular lesions in the ce- 
A
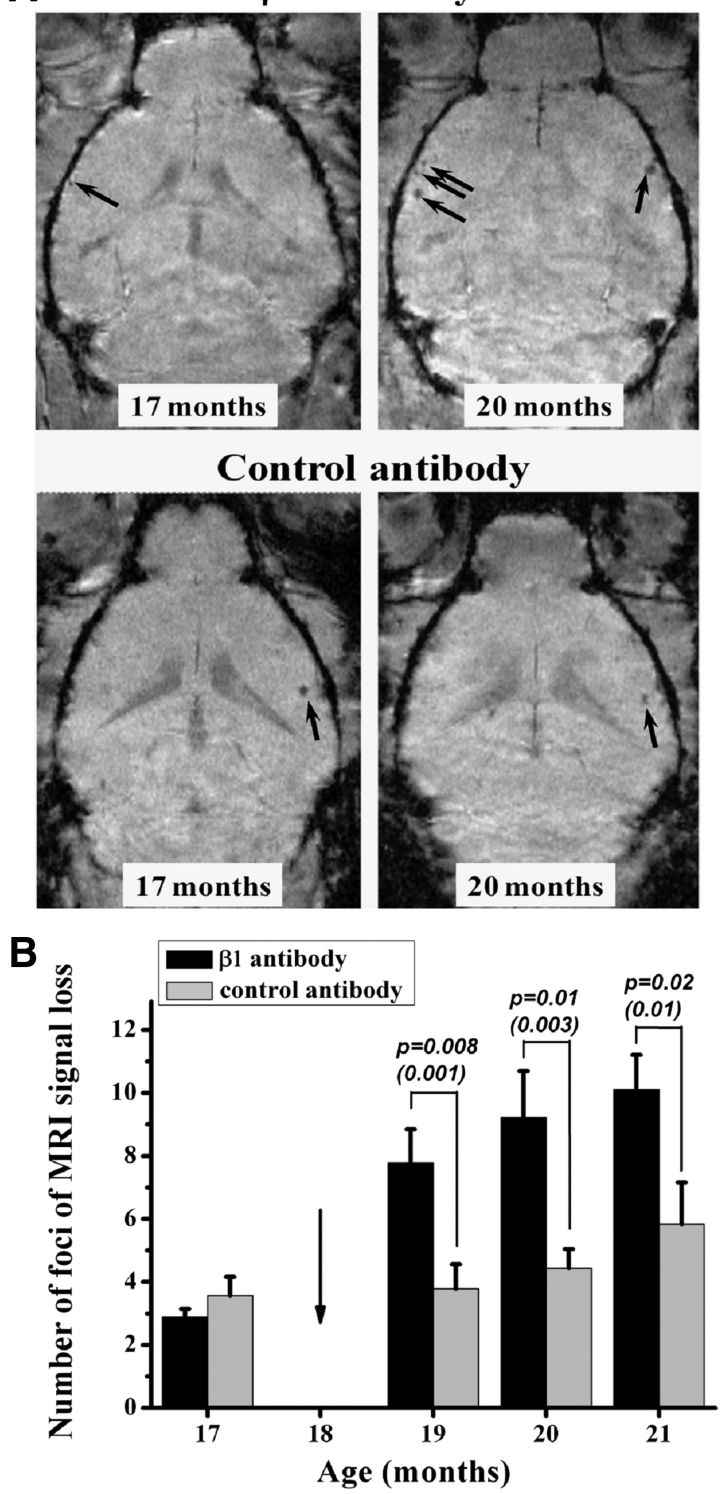

Figure 7. Immunization study with male APP23 mice. $\boldsymbol{A}, \mathrm{MR}$ images from two animals at baseline (17 months of age) and at 2 months after beginning of immunization ( 20 months of age). Additional sites of signal attenuation were detected only in the brain cortex of the mouse receiving the $\beta 1$ antibody (arrows). Both animals had received SPI0 $24 \mathrm{~h}$ before being imaged. $B$, Number of signal attenuation foci (means \pm SEM, $n=8-10$ mice in each group and time point) in cerebral cortex, $24 \mathrm{~h}$ after SPIO administration to immunized mice. Treatment with the $\beta 1$ antibody increased the number of foci with MRI signal attenuation. A baseline measurement was done at 17 months. The arrow indicates the beginning of the immunization, at 18 months of age. The significance levels $p$ correspond to Mann-Whitney comparisons at the specified time points and, in parentheses, to the longitudinal comparisons of the $\log 10$ of the individual counts using ANOVA with random effects.

rebral cortex of APP23 mice following $\beta 1$ antibody treatment. Our data demonstrate that MRI imaging is suited to detect such treatment-related alterations in mice. This includes minor lesions as these were primarily increased during treatment.

\section{Discussion}

In vivo detection of microvascular pathologies such as microhemorrhages and vasculitis in humans gains increasing importance as these lesions potentially contribute to cognitive dysfunction or indicate a risk of hemorrhagic stroke. Moreover, microhemorrhages may be elevated by $\mathrm{A} \beta$ immunotherapy, various ap-
Table 2. Summary of histological analysis performed on the cerebral cortex from 6 APP23 male mice treated with the $\beta 1$ antibody and on 5 male APP23 mice that had received the control antibody

\begin{tabular}{lccl}
\hline Parameter & Control antibody & $\beta 1$ antibody & Statistics \\
\hline Blood vessels with CAA & $25.6 \pm 3.8$ & $33.9 \pm 3.3$ & $p=0.04$ \\
$\begin{array}{l}\text { Areas with iron containing } \\
\quad \text { macrophages (total) }\end{array}$ & $3.6 \pm 1.2$ & $15.2 \pm 3.0$ & $p=0.03$ \\
$\begin{array}{l}\text { By severity grade } \\
\text { Low: } 1-3 \text { macrophages }\end{array}$ & & & \\
$\quad$ Moderate: $4-10$ macrophages & $2.5 \pm 0.9$ & $7.1 \pm 1.6$ & $p=0.006$ \\
$\quad$ Abundant: $>10$ macrophages & $0.3 \pm 0.2$ & $1.4 \pm 0.5$ & $p=0.01$ \\
Amyloid vessels with iron-containing & $2.6 \pm 0.7$ & $3.0 \pm 0.5$ & n.s. \\
$\quad$ macrophages & & &
\end{tabular}

Numbers (means \pm SEM) refer to counts of areas in the vicinity of CAA vessels presenting iron-containing macrophages, averaged over 11 sections per animal.

proaches of which are currently developed for $\mathrm{AD}$. In patients, focal hypointensities have been detected using $\mathrm{T}_{2}{ }^{*}$-weighted gradient-echo protocols (Fazekas et al., 1999; Tanaka et al., 1999; Imaizumi et al., 2004; Pettersen et al., 2008; Ayaz et al., 2010). While the signal attenuation is expected to result from the paramagnetic properties of the hemoglobin degradation product hemosiderin, the relationship to histologically detected microhemorrhages could not be studied satisfactorily in humans. In the present study, we have demonstrated that MRI can detect microvascular pathologies in a CAA-containing mouse model of $\mathrm{AD}$ and that an increase during $\mathrm{A} \beta$ immunotherapy can be followed in vivo.

$\mathrm{T}_{2}{ }^{*}$-weighted gradient-echo images acquired from the brain of aged APP23 mice without contrast agent showed only a small number of foci of signal loss in cortical and thalamic areas, which resulted from hemosiderin in macrophages as confirmed by Berlin blue histological staining. After administration of SPIO, $24 \mathrm{~h}$ before the measurements, the hypointense foci identified in precontrast images could be enhanced considerably and many more sites of signal loss became clearly apparent. Histology at the sites of signal loss demonstrated the presence of iron entrapped in macrophages in the vicinity of pathologic, CAA-affected blood vessels, or more rarely in the vessel wall. A fraction of the sites showed additional pathology such as thickened vessel walls and vasculitis. The same spectrum of lesions was found with or without SPIO treatment. Hence, sites of signal attenuation in the absence of labeling could pathologically not be distinguished from sites detected after SPIO labeling. This assertion is based on the analysis of histological slices obtained from the sites of attenuated signals as detected in vivo, rather than on a systematic slicing of whole brains. The number of cortical sites displaying signal attenuation increased with age. These observations suggest that administration of iron oxide nanoparticles improved the detection of microhemorrhages in the brains of APP23 mice and also led to the labeling of additional sites of microvascular alterations. Our findings match previous observations in the same transgenic line showing a very similar location of iron-containing macrophages (siderophages) at areas displaying cerebrovascular amyloid, additional vascular pathology at a subset of sites, and an age-related increase in these microhemorrhages (Calhoun et al., 1999; Winkler et al., 2001). Interestingly, at the sites analyzed in the present study, we did not detect any acute hemorrhages in agreement with their very rare occurrence in previous investigations (Calhoun et al., 1999; Winkler et al., 2001). The vast majority of the sites of signal attenuation, therefore, represent older lesions.

To further confirm that the sites of attenuated MRI signal in the APP23 brain reflected CAA-associated microvascular pathol- 
ogy, we introduced a strong presenilin mutation (APP23xPS45 mice), which shifted $\mathrm{A} \beta$ generation toward the 42 isoform, thus increasing parenchymal and reducing vascular amyloid (Herzig et al., 2004). Consistent with this change, MRI detected a much smaller number of attenuated signal sites than in APP23 mice. Very similar results were obtained with APP24 and APP51 mice, both of which develop significantly less CAA and microvascular pathology than APP23 mice. Overall, these observations strongly indicate that following administration of SPIO, MRI detects CAA-related pathology, and not changes reflecting deposition of parenchymal amyloid.

Different mechanisms may have contributed to the foci of signal attenuation detected by MRI after administration of contrast agent. (1) Since iron was found entrapped in macrophages, it is conceivable that SPIO nanoparticles were endocytosed in the blood circulation by macrophages, which then infiltrated the brain at sites of pathologic vessels. Although these sites coincided to a certain extent with the location of previous microhemorrhages, they also represented additional CAA-related microvasculopathy, characterized by the presence of macrophages (Vinters et al., 1998; Thal et al., 2008). Circulating macrophages have been shown to migrate from the lumen into the vessel wall (Vinters et al., 1998) and may be recruited into amyloidcontaining AD or APP transgenic mouse brain (Fiala et al., 2002; Stalder et al., 2005; Simard et al., 2006). Macrophages are able to transmigrate the blood-brain barrier $(\mathrm{BBB})$ when attracted by chemokines produced by amyloid $\beta$-stimulated cells (Fiala et al., 1998; El Khoury et al., 2007; Hickman and El Khoury, 2010). (2) A partial, local BBB breakdown could also have caused leakage of contrast agent, which would then be taken up by microglia in brain. However, studies in old APP23 mice with the extracellular contrast agent, gadolinium-tetraazacyclododecanetetraacetic acid (Gd-DOTA), administered intravenously did not show significant hyperintense signals, indicating that the agent did not leak through the BBB (data not shown). With a mean molecular diameter of $0.9 \mathrm{~nm}$, Gd-DOTA is considerably smaller than the SPIO nanoparticles (mean diameter of $150 \mathrm{~nm}$ ) used in this study (Corot et al., 2003). These results are in agreement with histological analyses performed by Winkler et al. (2001), demonstrating an absence of significant BBB disruption in old APP23 animals.

The present investigation also demonstrates that SPIOenhanced MRI is well suited to detect the treatment-induced elevation of microhemorrhage in mice as observed in some studies of $\mathrm{A} \beta$ immunotherapy (Pfeifer et al., 2002; Wilcock et al., 2007). While chronically treated with the $\mathrm{A} \beta$ antibody $\beta 1$, APP23 mice showed an increase in the number of sites with attenuated MRI signals, which could be followed with time. Lack of a change in control antibody-treated APP23 mice and $\beta 1$-treated wildtype animals demonstrated the specificity of the measurements. Postmortem histological analysis confirmed a corresponding increase in the number of CAA-associated iron-containing macrophages in brain. These results are in agreement with previous studies of $\beta 1$-treated aged APP23 mice demonstrating a twofold increase in the frequency and severity of microhemorrhages (Pfeifer et al., 2002; Burbach et al., 2007). Overall, our in vivo and postmortem observations show an increase in CAA-related microvascular pathology in the cerebral cortex of APP23 mice as a consequence of passive $\mathrm{A} \beta$ immunotherapy. They demonstrate the sensitivity of the MRI method used to detect this increase in living animals. As SPIO is approved for clinical use, the approach has translational value and can be used to screen or to monitor patients for microvascular lesions.
In conclusion, the present results demonstrate that MRI in combination with the administration of SPIO can be used noninvasively to study CAA-related microvascular lesions in transgenic mouse models of $\mathrm{AD}$. The technique is complementary to MR angiography, which has detected flow disturbances at the level of the circle of Willis in old APP23 mice (Beckmann et al., 2003; Thal et al., 2009). Overall, these approaches visualize the loss of vascular integrity, which provides the basis for the agerelated impairment of the cerebral blood volume response to pharmacological or electrical stimulation shown in brain functional MRI studies in APP23 mice (Mueggler et al., 2003). These observations support the idea that cerebral microcirculatory abnormalities developing progressively contribute to AD pathogenesis and cognitive impairment (Meyer et al., 2008). Although not well understood, certain forms of $A \beta$ immunotherapy may have the potential to enhance such pathologies, which could be monitored noninvasively by an approach as described here.

\section{References}

Abramowski D, Wiederhold KH, Furrer U, Jaton AL, Neuenschwander A, Runser MJ, Danner S, Reichwald J, Ammaturo D, Staab D, Stoeckli M, Rueeger H, Neumann U, Staufenbiel M (2008) Dynamics of A $\beta$ turnover and deposition in different APP transgenic mouse models following gamma-secretase inhibition. J Pharmacol Exp Ther 327:411-424.

Ayaz M, Boikov AS, Haacke EM, Kido DK, Kirsch WM (2010) Imaging cerebral microbleeds using susceptibility weighted imaging: one step toward detecting vascular dementia. J Magn Reson Imaging 31:142-148.

Beckmann N, Schuler A, Mueggler T, Meyer EP, Wiederhold KH, Staufenbiel M, Krucker T (2003) Age-dependent cerebrovascular abnormalities and blood flow disturbances in APP23 mice modeling Alzheimer's disease. J Neurosci 23:8453-8459.

Beckmann N, Cannet C, Babin AL, Blé FX, Zurbruegg S, Kneuer R, Dousset V (2009) In vivo visualization of macrophage infiltration and activity in inflammation using MRI. Wiley Interdiscip Rev Nanomed Nanobiotechnol 1:272-298.

Boche D, Zotova E, Weller RO, Love S, Neal JW, Pickering RM, Wilkinson D, Holmes C, Nicoll JA (2008) Consequence of A\{beta $\}$ immunization on the vasculature of human Alzheimer's disease brain. Brain 131: 3299-3310.

Bodendorf U, Danner S, Fischer F, Stefani M, Sturchler-Pierrat C, Wiederhold KH, Staufenbiel M, Paganetti P (2002) Expression of human beta-secretase in the mouse brain increases the steady-state level of beta-amyloid. J Neurochem 80:799-806.

Brody DL, Holtzman DM (2008) Active and passive immunotherapy for neurodegenerative disorders. Annu Rev Neurosci 31:175-193.

Burbach GJ, Vlachos A, Ghebremedhin E, Del Turco D, Coomaraswamy J, Staufenbiel M, Jucker M, Deller T (2007) Vessel ultrastructure in APP23 transgenic mice after passive anti-Abeta immunotherapy and subsequent intracerebral hemorrhage. Neurobiol Aging 28:202-212.

Busche MA, Eichhoff G, Adelsberger H, Abramowski D, Wiederhold KH, Haass C, Staufenbiel M, Konnerth A, Garaschuk O (2008) Clusters of hyperactive neurons near amyloid plaques in a mouse model of Alzheimer's disease. Science 321:1686-1689.

Calhoun ME, Burgermeister P, Phinney AL, Stalder M, Tolnay M, Wiederhold KH, Abramowski D, Sturchler-Pierrat C, Sommer B, Staufenbiel M, Jucker M (1999) Neuronal overexpression of mutant amyloid precursor protein results in prominent deposition of cerebrovascular amyloid. Proc Natl Acad Sci U S A 96:14088-14093.

Cordonnier C, Al-Shahi Salman R, Wardlaw J (2007) Spontaneous brain microbleeds: systematic review, subgroup analyses and standards for study design and reporting. Brain 130:1988-2003.

Corot C, Violas X, Robert P, Gagneur G, Port M (2003) Comparison of different types of blood pool agents (P792, MS325, USPIO) in a rabbit MR angiography-like protocol. Invest Radiol 38:311-319.

El Khoury J, Toft M, Hickman SE, Means TK, Terada K, Geula C, Luster AD (2007) Ccr2 deficiency impairs microglial accumulation and accelerates progression of Alzheimer-like disease. Nat Med 13:432-438.

Fazekas F, Kleinert R, Roob G, Kleinert G, Kapeller P, Schmidt R, Hartung HP (1999) Histopathologic analysis of foci of signal loss on gradient-echo T2*-weighted MR images in patients with spontaneous intracerebral 
hemorrhage: evidence of microangiopathy-related microbleeds. AJNR Am J Neuroradiol 20:637-642.

Fiala M, Zhang L, Gan X, Sherry B, Taub D, Graves MC, Hama S, Way D, Weinand M, Witte M, Lorton D, Kuo YM, Roher AE (1998) Amyloidbeta induces chemokine secretion and monocyte migration across a human blood-brain barrier model. Mol Med 4:480-489.

Fiala M, Liu QN, Sayre J, Pop V, Brahmandam V, Graves MC, Vinters HV (2002) Cyclooxygenase-2-positive macrophages infiltrate the Alzheimer's disease brain and damage the blood-brain barrier. Eur J Clin Invest 32:360-371.

Greenberg SM, Gurol ME, Rosand J, Smith EE (2004) Amyloid angiopathyrelated vascular cognitive impairment. Stroke 35:2616-2619.

Herzig MC, Winkler DT, Burgermeister P, Pfeifer M, Kohler E, Schmidt SD, Danner S, Abramowski D, Stürchler-Pierrat C, Bürki K, van Duinen SG, Maat-Schieman ML, Staufenbiel M, Mathews PM, Jucker M (2004) Abeta is targeted to the vasculature in a mouse model of hereditary cerebral hemorrhage with amyloidosis. Nat Neurosci 7:954-960.

Hickman SE, El Khoury J (2010) Mechanisms of mononuclear phagocyte recruitment in Alzheimer's disease. CNS Neurol Disord Drug Targets 9:168-173.

Imaizumi T, Horita Y, Chiba M, Hashimoto Y, Honma T, Niwa J (2004) Dot-like hemosiderin spots on gradient echo $\mathrm{T} 2{ }^{*}$-weighted magnetic resonance imaging are associated with past history of small vessel disease in patients with intracerebral hemorrhage. J Neuroimaging 14:251-257.

Jellinger KA (2002) Alzheimer disease and cerebrovascular pathology: an update. J Neural Transm 109:813-836.

Jung CW (1995) Surface properties of superparamagnetic iron oxide MR contrast agents: ferumoxides, ferumoxtran, ferumoxsil. Magn Reson Imaging 13:675-691.

Kinnecom C, Lev MH, Wendell L, Smith EE, Rosand J, Frosch MP, Greenberg SM (2007) Course of cerebral amyloid angiopathy-related inflammation. Neurology 68:1411-1416.

Maat-Schieman ML, van Duinen SG, Rozemuller AJ, Haan J, Roos RA (1997) Association of vascular amyloid beta and cells of the mononuclear phagocyte system in hereditary cerebral hemorrhage with amyloidosis (Dutch) and Alzheimer disease. J Neuropathol Exp Neurol 56:273-284.

Meyer EP, Ulmann-Schuler A, Staufenbiel M, Krucker T (2008) Altered morphology and 3D architecture of brain vasculature in a mouse model for Alzheimer's disease. Proc Natl Acad Sci U S A 105:3587-3592.

Mueggler T, Baumann D, Rausch M, Staufenbiel M, Rudin M (2003) Agedependent impairment of somatosensory response in the amyloid precursor protein 23 transgenic mouse model of Alzheimer's disease. J Neurosci 23:8231-8236.

Paganetti PA, Lis M, Klafki HW, Staufenbiel M (1996) Amyloid precursor protein truncated at any of the gamma-secretase sites is not cleaved to beta-amyloid. J Neurosci Res 46:283-293.
Pettersen JA, Sathiyamoorthy G, Gao FQ, Szilagyi G, Nadkarni NK, St George-Hyslop P, Rogaeva E, Black SE (2008) Microbleed topography, leukoaraiosis, and cognition in probable Alzheimer disease from the Sunnybrook dementia study. Arch Neurol 65:790-795.

Pfeifer M, Boncristiano S, Bondolfi L, Stalder A, Deller T, Staufenbiel M, Mathews PM, Jucker M (2002) Cerebral hemorrhage after passive antiAbeta immunotherapy. Science 298:1379.

Simard AR, Soulet D, Gowing G, Julien JP, Rivest S (2006) Bone marrowderived microglia play a critical role in restricting senile plaque formation in Alzheimer's disease. Neuron 49:489-502.

Stalder AK, Ermini F, Bondolfi L, Krenger W, Burbach GJ, Deller T, Coomaraswamy J, Staufenbiel M, Landmann R, Jucker M (2005) Invasion of hematopoietic cells into the brain of amyloid precursor protein transgenic mice. J Neurosci 25:11125-11132.

Sturchler-Pierrat C, Abramowski D, Duke M, Wiederhold KH, Mistl C, Rothacher S, Ledermann B, Bürki K, Frey P, Paganetti PA, Waridel C, Calhoun ME, Jucker M, Probst A, Staufenbiel M, Sommer B (1997) Two amyloid precursor protein transgenic mouse models with Alzheimer disease-like pathology. Proc Natl Acad Sci U S A 94:13287-13292.

Tanaka A, Ueno Y, Nakayama Y, Takano K, Takebayashi S (1999) Small chronic hemorrhages and ischemic lesions in association with spontaneous intracerebral hematomas. Stroke 30:1637-1642.

Thal DR, Griffin WS, de Vos RA, Ghebremedhin E (2008) Cerebral amyloid angiopathy and its relationship to Alzheimer's disease. Acta Neuropathol 115:599-609.

Thal DR, Capetillo-Zarate E, Larionov S, Staufenbiel M, Zurbruegg S, Beckmann N (2009) Capillary cerebral amyloid angiopathy is associated with vessel occlusion and cerebral blood flow disturbances. Neurobiol Aging 30:1936-1948.

Vinters HV, Natté R, Maat-Schieman ML, van Duinen SG, Hegeman-Kleinn I, Welling-Graafland C, Haan J, Roos RA (1998) Secondary microvascular degeneration in amyloid angiopathy of patients with hereditary cerebral hemorrhage with amyloidosis, Dutch type (HCHWA-D). Acta Neuropathol 95:235-244.

Weissleder R, Elizondo G, Wittenberg J, Rabito CA, Bengele HH, Josephson L (1990) Ultrasmall superparamagnetic iron oxide: characterization of a new class of contrast agents for MR imaging. Radiology 175:489-493.

Wilcock DM, Jantzen PT, Li Q, Morgan D, Gordon MN (2007) Amyloidbeta vaccination, but not nitro-nonsteroidal anti-inflammatory drug treatment, increases vascular amyloid and microhemorrhage while both reduce parenchymal amyloid. Neuroscience 144:950-960.

Winkler DT, Bondolfi L, Herzig MC, Jann L, Calhoun ME, Wiederhold KH, Tolnay M, Staufenbiel M, Jucker M (2001) Spontaneous hemorrhagic stroke in a mouse model of cerebral amyloid angiopathy. J Neurosci 21: $1619-1627$. 